\title{
A Random Tree Model Associated with Random Graphs
}

\author{
David Aldous* \\ Department of Statistics, University of California, Berkeley CA 94720
}

\begin{abstract}
Grow a tree on $n$ vertices by starting with no edges and successively adding an edge chosen uniformly from the set of possible edges whose addition would not create a cycle. This process is closely related to the classical random graph process. We describe the asymptotic structure of the tree, as seen locally from a given vertex. In particular, we give an explicit expression for the asymptotic degree distribution. Our results an be applied to study the random minimum-weight spanning tree question, when the edge-weight distribution is allowed to vary almost arbitrarily with $n$.
\end{abstract}

\section{INTRODUCTION}

The construction indicated in the abstract, and stated more formally in Section 2 , yields a certain random tree $\mathscr{T}_{n}$ on $n$ vertices. It is easy to calculate that, for the star graph $t_{n}$ centered at vertex 1 ,

$$
P\left(\mathscr{T}_{n}=t_{n}\right)=2^{n-1}(n-1) ! /(2 n-2) !
$$

and so for $n \geq 4, \mathscr{T}_{n}$ is not the uniform random labeled tree (for which the probability is $1 / n^{n-2}$ ). Other explicit calculations are harder. The substance of this paper is Theorem 1 below, which gives information about certain aspects of the asymptotic behavior of $\mathscr{T}_{n}$ as $n \rightarrow \infty$.

Associate with $\mathscr{T}_{n}$ the random fringe subtree $\mathscr{F}_{n}$ defined as follows. Regard $\mathscr{T}_{n}$ as rooted at some prespecified vertex (1, say). Each edge from 1 leads to some

*Research supported by N.S.F. Grant MCS87-01426.

Random Structures and Algorithms, Vol. 1, No. 4 (1990)

(C) 1990 John Wiley \& Sons, Inc. CCC 1042-9832/90/040383-20\$04.00 
subtree, whose combined sizes (numbers of vertices) equal $n-1$. Delete the subtree of maximal size (if not unique, choose arbitrarily). Let $\mathscr{F}_{n}$ be the remaining tree, rooted at 1 . By removing labels we may regard $\mathscr{F}_{n}$ as a random element of the set $T$ of finite rooted unlabeled trees. ( $T$ includes the trivial tree consisting of a root only.)

Theorem 1. There exists a probability distribution $\pi$ on $T$ such that

Moreover

$$
P\left(\mathscr{F}_{n}=t\right) \rightarrow \pi(t) \text { as } n \rightarrow \infty ; t \in T
$$

$$
\sum_{t} \pi(t) \text { root-degree }(t)=1
$$

The limit $\pi$ is the distribution of a certain random tree $\mathscr{H}_{x}^{0}$, which we describe verbally now (and formally in Section 2). Write $\mathscr{G}_{s}$ for the family tree of a Galton-Watson branching process with 1 progenitor and Poisson(s) offspring distribution, considered as a rooted unlabeled tree. Construct a random tree process $\left(\mathscr{H}_{s}: 0 \leq s<\infty\right)$ as follows. $\mathscr{H}_{0}$ is a root only. During time $[s, s+d s]$, each vertex $v$ of $\mathscr{H}_{s}$ has chance $d s$ to have attached to it an independent copy of $\mathscr{G}_{s}$, if the realization of $\mathscr{G}_{s}$ is finite: if infinite, nothing is attached, but at the first time that some infinite tree is attempted the vertex $v$ at which it occurs is distinguished. This construction yields a finite tree $\mathscr{H}_{x}$ with one distinguished vertex. Delete the branch containing the distinguished vertex (if it is not the root): the remaining tree is $\mathscr{H}_{\nsim}^{0}$.

The proof is based upon the simple fact that sparse random graphs can be approximated locally by Galton-Watson trees. To indicate briefly the proof, consider the random graph process $\mathscr{G}\left(n, \frac{s}{n-1}\right)$, regarding $s$ as "time," and along with this construct the forest-valued process $\mathscr{T}_{n}(s)$ which evolves as $\mathscr{G}\left(n, \frac{s}{n-1}\right)$ except that an edge is added only if it does not create a cycle. Thus $\mathscr{T}_{n}$ is the end-result of the process $\left(\mathscr{T}_{n}(s) ; 0 \leq s \leq n-1\right)$. Consider the component of $\mathscr{T}_{n}(s)$ containing vertex 1 . For large $n$ this evolves like the process $\mathscr{H}_{s}$, the "distinguished vertex" being the vertex at which it joins the giant component. Thus the fringe of $\mathscr{T}_{n}(s)$ looks like the subtree of $\mathscr{H}_{s}$ at vertex 1 (where $\mathscr{H}_{s}$ is considered rooted at the distinguished vertex), so the fringe of $\mathscr{T}_{n}$ looks like the subtree of $\mathscr{H}_{x}$, and this is $\mathscr{H}_{x}^{0}$. Our proofs in Sections 2 and 3 formalizing these process approximations are conceptually straightforward, though a little lengthy.

Theorem 1 is intended as a worked example in the general theory of asymptotic fringe distributions introduced in [1]. For any family of random trees one can define $\mathscr{F}_{n}$ as above (using an initial random root: in the present example, by symmetry this is equivalent to making vertex 1 the root). In most well-studied families it is easy to show the existence of a limit distribution $\pi$ as in Theorem 1 , or a related limit cycling behavior. For example, for the uniform random labeled tree, the limit distribution analogous to $\pi$ is just $\mathscr{G}_{1}$. The point of a result like Theorem 1 is that it implies convergence (to a limit defined in terms of $\pi$ ) of all functionals of the random tree which involve only "Jocal" structure. This contrasts with the traditional analytic techniques in combinatorics, which treat only one functional at a time. Propositions 2 and 3 below give specializations of Theorem 1 to more concrete questions. 
Write $D(n)+1$ for the degree of vertex 1 in $\mathscr{T}_{n}$. Theorem 1 implies $D(n) \stackrel{d}{\rightarrow} D(\infty)$, where $D(\infty)$ is the degree of the root of $\mathscr{H}_{x}^{0}$, and one can calculate this limit distribution using the description of the tree-process $\mathscr{H}_{s}^{0}$.

\section{Proposition 2.}

$$
P(D(\infty)=i)=\int_{0}^{1} e^{-\Phi(u)}(\Phi(u))^{i} / i ! d u ; \quad i \geq 0
$$

where

$$
\Phi(u)=\int_{0}^{u} \frac{\log 1 / x}{1-x} d x, \quad u<1 .
$$

The proof is given in Section 4.1. Similarly, Theorem 1 implies that the height and size of the subtree $\mathscr{F}_{n}$ converge to the height and size of $\mathscr{H}_{x}^{0}$, though we do not have any simple explicit expression for these limit distributions. (Note that although Proposition 2 gives the degree of the root as a mixture of Poissons, it is not true that $\pi$ is the corresponding mixture of $\left(\xi_{s}\right)$ ).

As a second application of Theorem 1, we get some deeper insight into the well-studied problem of random minimum-weight spanning trees. The results stated below will be proved in Section 4.4. Take the complete graph on $n$ vertices. Attach i.i.d. edge-weights $\eta_{i j}^{(n)}$, and consider

(a) the special case: $\hat{\eta}^{(n)}$ has uniform distribution on $(0, n-1)$;

(b) the general case: $\eta^{(n)} \geq 0$ has some continuous distribution function $G_{n}$, varying arbitrarily with $n$.

In the special case, the minimum-weight spanning tree (constructed using Kruskal's greedy algorithm) is exactly $\mathscr{T}_{n}$, and for each edge of $\mathscr{T}_{n}$ the weight is the time $s$ at which that edge was added in the tree-process $\left(\mathscr{T}_{n}(s) ; 0 \leq s \leq n-1\right)$. Write $W_{n}$ for the total weight of the minimum-weight spanning tree $\mathscr{T}_{n}$. A well-known result of Frieze [6] says that in the special case

$$
E W_{n} / n \rightarrow \zeta(3) \equiv \sum_{i=1}^{\infty} 1 / i^{3}
$$

Theorem 1 yields a stronger result on the asymptotic empirical distribution of edge-lengths of the minimum-weight spanning tree in the general case.

Proposition 3. Let $\theta_{n}$ be the weight of an edge chosen uniformly at random from the edges of $\mathscr{T}_{n}$. Then in the general case $n G_{n}\left(\theta_{n}\right) \stackrel{d}{\rightarrow} J$, where $0<J<\infty$ has density function $f_{J}$ defined by Equations (32) and (33) below.

Now $E W_{n}=(n-1) E \theta_{n}$, so under mild conditions we can deduce that $E W_{n} \sim n E G_{n}^{-1}(J / n)$. Here is the precise statement.

Corollary 4. Let $G_{n}^{-1}$ be the inverse distribution function of the edge-weights $\eta^{(1)}$. Suppose there exists $x_{0}<\infty$ such that for all $\alpha>0$ 


$$
\sup _{n} \int_{0}^{\infty} \frac{G_{n}^{-1}(x / n)}{G_{n}^{-1}\left(x_{0} / n\right)} e^{-\alpha x} d x<\infty
$$

Then as $n \rightarrow \infty$

$$
E W_{n} \sim n \int_{0}^{x} G_{n}^{-1}(x / n) f_{j}(x) d x
$$

A natural special case is where $G_{n} \equiv G$ does not depend on $n$ and satisfies

$$
G(x) \sim \gamma x^{\rho} \text { as } x \rightarrow 0 \quad(\gamma, \rho>0)
$$

Then

$$
E W_{n} \sim n^{1-1 / \rho} \gamma^{-1 / \rho} \int_{0}^{x} \frac{1}{2} x^{1 / \rho} f_{J}(x) d x
$$

and a calculus exercise (Section 4.3) shows the integral term is equal to

$$
\frac{1}{\rho} \sum_{i=1}^{\infty} \frac{(i-1+1 / \rho) !}{i !(i+1)^{2+1 / p}}
$$

Putting $\rho=1$, we recover $(1)$. The special case $(3,4)$ has been given by Timofeev [8] and by Avram and Bertsimas [3].

On a technical note, the continuity assumption on $G_{n}$ makes the statement of Proposition 3 simple, but is not in itself essential. The condition (2) excludes examples such as

$$
P\left(\eta^{(n)}=0\right)=1-P\left(\eta^{(n)}=1\right)=p(n) \sim \frac{\sqrt{\log n}}{n}
$$

where the total weight $W_{n}$ is dominated by the weights of a vanishingly small proportion of edges.

In principle, Theorem 1 could be applied to more general "cost" functionals associated with the random minimum spanning tree. Such functionals occur, for instance, in the context of set union-find algorithms $[5,7,9]$. But we have not pursued this topic.

\section{MAIN PART OF PROOF}

In this section we give the proof of Theorem 1, deferring until Section 3.3 a key lemma which requires some technical background (Section 3 ).

For each edge $(i, j)$ of the complete graph on $n$ vertices, create a real-valued random variable $Z_{i, j}$ distributed uniformly on $[0, n-1]$, independent for different edges. For each $s \in[0, n-1]$ let $\mathscr{G}(n, s /(n-1))$ be the random graph consisting of those edges $(i, j)$ with $Z_{i, j} \leq s$. Each edge has chance $s /(n-1)$ to be in this graph, and so we conform to the customary notation in the theory of random graphs. Regard $s$ as "time" and $s \rightarrow \mathscr{G}(n, s /(n-1))$ as a graph process, which 
adds new edges at random times $0<S_{1}<S_{2}<\cdots$. Associate with this process another process $\left(\mathscr{T}_{n}(s): 0 \leq s \leq n-1\right)$ using the rule:

when $\mathscr{G}(n, \cdot)$ adds a new edge $\left((i, j)\right.$, say) at time $S_{u}$, say, then $\mathscr{T}_{n}(\cdot)$ adds the same edge, provided this does not create a cycle.

It is clear that $\mathscr{T}_{n}(s)$ is a forest with the same connected components as $\mathscr{G}(n, s /(n-1))$. It is also clear that, given $\mathscr{T}_{n}(s)=t$, the next edge added is uniform on the set of allowable edges. So the final tree $\mathscr{T}_{n}(n-1) \equiv \mathscr{T}_{n}$, say, has the same distribution as the tree described in the abstract.

We now define another tree-valued process $\left(\mathscr{F}_{n}(s) ; 0 \leq s \leq n-1\right)$. Let $a(n) \rightarrow \infty, a(n) / n \rightarrow 0$ as $n \rightarrow \infty$ be constants specified later (Lemma 8 ). Write $\mathscr{B}(s)$ for the component of $\mathscr{T}_{n}(s)$ containing vertex 1 , considered as a rooted unlabeled tree with root 1 . Write $|t|$ for the size (number of vertices) of a tree $t$. Let

$$
L_{n}=\min \left\{s:\left|\mathscr{B}_{n}(s)\right|>a(n)\right\}
$$

At time $L_{n}$ some edge is added to $\mathscr{B}_{n}\left(L_{n}-\right)$ : write $V_{n}^{*}$ for the endpoint of that new edge in $\mathscr{B}_{n}\left(L_{n}-\right)$, and write $V_{n}^{* *}$ for the other endpoint. For $s<L_{n}$ define $\mathscr{F}_{n}(s)=\mathscr{B}_{n}(s)$. For $s \geq L_{n}$ define $\mathscr{F}_{n}(s)$ to be the subtree of $\mathscr{B}_{n}(s)$ consisting of all vertices $v$ for which the path from $v$ to 1 does not use the edge $\left(V_{n}^{*}, V_{n}^{* *}\right)$ added at time $L_{n}$. In this case $\left(s \geq L_{n}\right)$, regard $V_{n}^{*}$ as a distinguished vertex of $\mathscr{F}_{n}(s)$.

Formally, regard $\mathscr{F}_{n}(s)$ as taking values in the set $T \cup T^{*}$, where $T^{*}$ is the set of finite rooted trees with one distinguished vertex $v^{*}$ (which may be the root).

The main part of the result is

\section{Lemma 5.}

$$
\mathscr{F}_{n}(n-1) \stackrel{d}{\rightarrow} \mathscr{H}_{x},
$$

where $\mathscr{H}_{x}$ is the $T^{*}$-valued random tree defined following Equation (18) below.

Granted this result, we proceed as follows. For a tree $t^{*} \in T^{*}$, define a tree $t^{0} \in T$ as follows. If the distinguished vertex $v^{*}$ of $t$ is the root, then let $t^{0}=t$ (with the root undistinguished). If not, then $v^{*}$ is in one of the branches of $t$ (i.e., one of the subtrees rooted at a neighbor of the root of $t$ ): define $t^{0}$ to be $t$ minus the branch containing the distinguished vertex. $\mathscr{H}_{x}$.

Write $\mathscr{F}_{n}^{0}(n-1)$ and $\mathscr{H}_{x}^{0}$ for the trees obtained in this way from $\mathscr{F}_{n}(n-1)$ and

It is clear from the construction that $\mathscr{F}_{n}^{0}(n-1)$ is the same as the fringe tree $\mathscr{F}_{n}$ provided that $\left|\mathscr{F}_{n}(n-1)\right|<n / 2$. So

$$
P\left(\mathscr{F}_{n} \neq \mathscr{F}_{n}^{0}(n-1)\right) \leq P\left(\left|\mathscr{F}_{n}(n-1)\right| \geq n / 2\right) .
$$

By Lemma 5 and finiteness of $\mathscr{H}_{x}$, the bound tends to 0 as $n \rightarrow \infty$. Now Lemma 5 implies $\mathscr{F}_{n}^{0}(n-1) \stackrel{d}{\rightarrow} \mathscr{H}_{x}^{0}$, and so

$$
\mathscr{F}_{n} \stackrel{d}{\rightarrow} \mathscr{H}_{x}^{0}
$$

This is the convergence assertion of Theorem 1. The second assertion of the Theorem will be established in Section 4.1. 
We now start to work toward the definition of the limit $\mathscr{H}_{\infty}$ appearing in Lemma 5 . For $0 \leq s<\infty$ let $\mathscr{G}_{s}$ be the Galton-Watson family tree with 1 progenitor and with Poisson(s) offspring distribution. Regard $\mathscr{G}_{s}$ as a random element of $\hat{T}$, the set of rooted unlabeled finite or infinite trees. Write $\pi_{s}$ for the distribution of $\xi_{s}$.

Write $F(s)$ for the chance that the Galton-Watson tree $\mathscr{G}_{s}$ is finite. It is elementary and well-known that

$$
\begin{gathered}
F(s)=1, s \leq 1 \\
F(s)=\exp (s(F(s)-1)), \quad s>1 .
\end{gathered}
$$

The series solution

$$
F(s)=s^{-1} \sum_{i \geq 1} i^{i-1}\left(s e^{-s}\right)^{i} / i !, \quad s>1
$$

is not very useful: calculations are better done with the inverse function

$$
F^{-1}(u)=\frac{\log u}{u-1}, \quad 0<u<1 .
$$

It is well-known and elementary that

$$
E\left|\mathscr{G}_{s}\right|=1 /(1-s), \quad s<1
$$

and that the conditional distributions satisfy

$$
\operatorname{dist}\left(\mathscr{G}_{s} \| \mathscr{G}_{s} \mid<\infty\right)=\operatorname{dist}\left(\mathscr{G}_{\xi}\right)
$$

where $\hat{s}=s$ for $0 \leq s \leq 1$ and

$$
\hat{s}=s F(s)<1 \text { for } s>1 .
$$

For future reference, using (7) it is not hard to show

$$
s F(s) \rightarrow 0 \text { as } s \rightarrow \infty ; \int_{0}^{\infty} F(s) d s<\infty
$$

Given $s_{1}<s_{2}$, there is a natural construction of a joint distribution $\left(\mathscr{G}_{s_{1}}, \mathscr{G}_{s_{2}}\right)$ with the right marginals. Start with $\mathscr{G}_{s_{2}}$. Delete or retain each edge independently, with chance $s_{1} / s_{2}$ of retention. Then let $\mathscr{G}_{s_{1}}$ be the tree-component in the retained graph rooted at the original root: it is easy to verify that this $\mathscr{G}_{s_{1}}$ is indeed distributed as the Galton-Watson tree.

It is not hard to show that we can produce a $\hat{T}$-valued process $\left(\mathscr{G}_{s} ; 0 \leq s<\infty\right)$ which is a continuous-time nonhomogeneous Markov process, with the twodimensional distributions specified above. The evolution of this process as $s$ increases can be described in words as follows. In time $[s, s+d s]$, each vertex $v$ of $\xi_{s}$ has chance $d s$ to have a subtree appended to it, and such a subtree has distribution $\pi_{s}$, i.e., the distribution of the Galton-Watson (Poisson(s)) tree itself. 

is

Such a process is specified by its transition rate matrix $R_{s}$, whose interpretation

$$
R_{s}(t, u) d s=P\left(\mathscr{G}_{s+d s}=u \mid \mathscr{G}_{s}=t\right) ; t, u \in \hat{T}, t \neq u
$$

For our process we can specify $R_{s}$ as follows. For $t, t_{1} \in \hat{T}$ and $v$ a vertex of $t$, write $\left\langle t, v, t_{1}\right\rangle$ for the tree obtained by appending to $t$ a subtree rooted at $v$ which is a copy of $t_{1}$. (That is, connect $t$ and $t_{1}$ via a new edge from $v$ to the root of $t_{1}$, and regard the root of $t$ as the root of the new tree.)

Then

$$
R_{s}(t, u)=\sum_{v \in t} \sum_{s_{1} \in \hat{T}} 1_{\left(\left\langle t, v, t_{1}\right)=u\right)} \pi_{s}\left(t_{1}\right)
$$

(Technical aside. We are abusing notation here, because $\hat{T}$ is uncountable. Rather than set up $\mathscr{G}_{s}$ as a general-state-space Markov process, it is simpler to justify the arguments here by the truncation idea in Section 5.)

In terms of the process $\left(\mathscr{G}_{s} ; 0 \leq s<\infty\right)$, there is a random time $L$ at which the size of the tree becomes infinite:

$$
L=\inf \left\{\mathrm{s}:\left|\boldsymbol{G}_{\mathrm{s}}\right|=\infty\right\}
$$

Then

$$
P(L \leq s)=P\left(\left|\mathscr{G}_{s}\right|=\infty\right)=1-F(s)=\bar{F}(s), \text { say } .
$$

Then $1<L<\infty$ a.s., and the usual convention about making Markov processes right-continuous gives $\left|\mathscr{G}_{L}\right|=\infty$. A priori, it might happen that the left limit $\mathscr{G}_{L-}$ is an infinite tree, but it turns out that in fact this limit tree is a.s. finite. In other

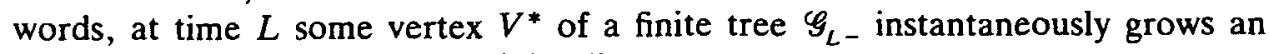
infinite tree. To argue this, (12) implies

$$
P\left(L \in[s, s+d s] \mid \mathscr{G}_{s}=t\right)=|t| \tilde{F}(s) d s, \quad t \in T
$$

and so

$$
P\left(\mathscr{G}_{L_{-}}=t, L \in[s, s+d s]\right)=|t| P\left(\mathscr{G}_{s}=t\right) \bar{F}(s) d s
$$

Thus

$$
P\left(\left|\mathscr{G}_{L-}\right|<\infty, L \in[s, s+d s]\right)=b(s) \bar{F}(s) d s
$$

where

$$
\begin{aligned}
b(s) & =\sum_{t \in r}|t| P\left(\mathscr{G}_{s}=t\right) \\
& =E\left|\mathscr{G}_{s}\right| 1_{\left(\left|\Im_{s}\right|<x\right)} \\
& =F(s) E\left|\mathscr{G}_{s}\right| \text { by }(9) \\
& =\frac{F(s)}{1-s F(s)} \text { by }(8) .
\end{aligned}
$$


To verify $\left|\mathscr{G}_{L-}\right|<\infty$ a.s. it suffices, by (13) and (16), to show

$$
\bar{F}(d s)=b(s) \bar{F}(s) d s
$$

and this follows analytically from $(6,17)$.

We now define a process $\left(\mathscr{H}_{s}\right)$ associated with $\left(\mathscr{G}_{s}\right)$. The process $\mathscr{G}_{s}$ evolves by vertices growing subtrees, which may be finite or infinite subtrees. The process $\mathscr{H}_{s}$ evolves in the same way, except that the infinite subtrees are censored: also, the vertex $V^{*}$ at which the first infinite subtree in $\mathscr{G}$ is grown at time $L$ is distinguished in $\mathscr{H}_{s}, s \geq L$. In other words,

$$
\begin{aligned}
& \mathscr{H}_{s}=\mathscr{G}_{s}, s<L \\
& \mathscr{K}_{L}=\mathscr{G}_{L_{-}}, \text {with } V^{*} \text { distinguished }
\end{aligned}
$$

and after time $L$ the process $\mathscr{H}_{s}$ evolves as the Markov process with transition rates $R_{s}$ given by (12), except that the sum is over $t_{1} \in T$ instead of $\hat{T}$.

The process $\left(\mathscr{H}_{s} ; 0 \leq s<\infty\right)$ takes values in $T \cup T^{*}$ (recall $T^{*}$ indicates finite trees with a distinguished vertex). The limit $\mathscr{H}_{x}$ certainly exists: we have to show

$$
\left|\mathscr{H}_{x}\right|<\infty \text { a.s. }
$$

To show this, condition on $L=s_{0}>1$ and $\left|\mathscr{H}_{L}\right|=a_{0}$. Then the conditional expected size $a(s)=E\left|\mathscr{H}_{s}\right|, s>s_{0}$ satisfies

$$
a^{\prime}(s)=a(s) b(s)
$$

where $b(s)=E\left|\mathscr{G}_{s}\right| 1_{\left(\left|\mathcal{S}_{s}\right|<x\right)}=F(s) /(1-s F(s))$, as at (17). But (11) implies $\int^{\infty} b(s) d s<\infty$, and so

$$
a(\infty)=a\left(s_{0}\right) \exp \left(\int_{s_{0}}^{\infty} b(s) d s\right)<\infty
$$

establishing finiteness of $\left|\mathscr{H}_{x}\right|$.

Lemma 5 now makes sense. Let us show that its proof can be reduced to the proof of

Lemma 6. For fixed $s<\infty$,

$$
\mathscr{F}_{n}(s) \stackrel{d}{\rightarrow} \mathscr{H}_{s} \text { as } n \rightarrow \infty .
$$

Given this result, to prove Lemma 5 we need only show

$$
\lim _{s \rightarrow x} \limsup _{n \rightarrow x} P\left(\mathscr{F}_{n}(n-1) \neq \mathscr{F}_{n}(s)\right)=0 \text {. }
$$

To argue this, recall that $L_{n}$ is the first time $s$ that vertex 1 enters a "giant component" (size $\geq a(n)$ ) of $\mathscr{G}\left(n, s /(n-1)\right.$ ). Write $q_{n}(s)=$ expected proportion of vertices outside the smallest giant component of $\mathscr{G}(n, s /(n-1))$. On $\left\{L_{n}<s\right\}$, 


$$
P\left(\mathscr{F}_{n}(s+d s) \neq \mathscr{F}_{n}(s) \mid \mathscr{G}(n, s /(n-1))\right) \leq \frac{d s}{n-1} \times\left|\mathscr{F}_{n}(s)\right|
$$

$\times$ number of vertices outside smallest giant component of $\mathscr{G}(n, s /(n-1))$.

So for any fixed $a$

$$
P\left(\mathscr{F}_{n}(s+d s) \neq \mathscr{F}_{n}(s),\left|\mathscr{F}_{n}(s)\right| \leq a, L_{n}<s\right) \leq \frac{n}{n-1} a q_{n}(s) d s
$$

So for $s>s_{0}$,

$$
P\left(\mathscr{F}_{n}(s+d s) \neq \mathscr{F}_{n}(s),\left|\mathscr{F}_{n}(s)\right| \leq a\left|L_{n}<s_{0},\right| \mathscr{F}_{n}\left(s_{0}\right) \mid \leq a\right) \leq \frac{n}{n-1} \frac{a q_{n}(s)}{1-h_{n}\left(s_{0}, a\right)} d s
$$

where

$$
h_{n}\left(s_{0}, a\right)=P\left(\left|\mathscr{F}_{n}\left(s_{0}\right)\right|>a\right)+P\left(L_{n}>s_{0}\right)
$$

Integrating over $s \geq s_{0}$,

$$
P\left(\mathscr{F}_{n}(n-1) \neq \mathscr{F}_{n}\left(s_{0}\right)\right) \leq h_{n}\left(s_{0}, a\right)+\frac{n}{n-1} \frac{a}{1-h_{n}\left(s_{0}, a\right)} \int_{s_{0}}^{\infty} q_{n}(s) d s .
$$

Now from Lemma 6 and finiteness of $\mathscr{H}_{x}$,

$$
\lim _{s_{0} \rightarrow \infty} \limsup _{n \rightarrow \infty} P\left(\left|\mathscr{F}_{n}\left(s_{0}\right)\right|>a\right)=0 .
$$

Moreover, $P\left(L_{n}>s\right) \leq q_{n}(s)$, so (19) follows from (20) and

$$
\lim _{s_{0} \rightarrow \infty} \limsup _{n \rightarrow x} \int_{s_{0}}^{\infty} q_{n}(s) d s=0 .
$$

This is Lemma $8(\mathrm{~b})$ below.

\section{TECHNICAL BACKGROUND}

\subsection{Random Graphs}

There is a well-developed theory of random graphs, treated in detail by Bollobas [4]. The facts we need are comparatively simple, though not quite of the standard form. We state them below, and sketch them briefly without going into routine details.

Recall $\pi_{s}(x)=P\left(\mathscr{G}_{s}=x\right)$ for a finite rooted unlabeled tree $x$. We can extend this to finite rooted graphs $x$ by putting $\pi_{1}(x)=0$ if $x$ is not a tree. Let $C_{?}^{\prime \prime}(x)$ denote the (random) proportion of vertices $i$ of $\mathscr{G}(n, s /(n-1))$ for which the component containing $i$, considered as a graph rooted at $i$, is isomorphic to $x$. 
Lemma 7. For each finite rooted graph $x$,

$$
\sup _{0 \leq s<\infty}\left|C_{s}^{n}(x)-\pi_{s}(x)\right| \stackrel{p}{\rightarrow} 0 \text { as } n \rightarrow \infty
$$

Sketch. The result for fixed $s$ is standard, by computing first and second moments $[4$, Exercise 4.6]. So for any finite set $G$ of rooted graphs

$$
\sum_{x \in G} C_{s}^{n}(x) \stackrel{p}{\rightarrow} \sum_{x \in G} \pi_{s}(x)
$$

Suppose the set $G$ is closed under passing to subgraphs (same root). Then both sides are decreasing in $s$, and the right side is continuous in $s$, so uniform convergence holds. The lemma follows by applying this fact to $G=\mathscr{S}[x]$ and $G=\mathscr{S}[x]-\{x\}$, where $\mathscr{S}[x]$ is the set of all subgraphs of $G$.

The second fact we need is that there exists a deterministic sequence $a(n) \rightarrow \infty$ such that the following lemma holds. Let $a_{1}(n) / a(n) \rightarrow 1$. Let $A_{s}^{n .1}\left[\right.$ resp. $A_{s}^{n .2}$ ] be the proportion of vertices of $\mathscr{G}(n, s /(n-1))$ in all components [resp. the smallest component] of size at least $a_{1}(n)$. Then

Lemma 8. (a) For $u=1,2$,

$$
\sup _{0 \leq s<x}\left|1-F(s)-A_{s}^{n, u}\right| \stackrel{p}{\rightarrow} 0 \text { as } n \rightarrow \infty \text {. }
$$

(b) $\lim _{s_{0} \rightarrow \infty} \lim \sup _{n} \int_{s_{0}}^{\infty}\left(1-E A_{s}^{n .2}\right) d s=0$.

Sketch. It is easy to see that $E A_{s_{n}}^{n, 1} \rightarrow 0$ as $s_{n} \downarrow 1$; indeed this follows from Lemma 7 , since $\Sigma_{x} \pi_{1}(x)=1$. Also $F(s) \rightarrow 1$ as $s \downarrow 1$, so to prove (a) it suffices to fix $s_{0}>1$ and prove the assertion with the sup taken over $s \geq s_{0}$. But this, and (b), are essentially consequences of deeper results in Ref. 4, Section 6.3.

\subsection{Convergence of Finite-state Processes}

The final technical ingredient is a lemma on convergence of non-Markov processes to a Markov limit. Let $(X(s): 0 \leq s<\infty)$ be a nonhomogeneous Markov process on a finite state-space $T$ with transition rates $R_{s}\left(t_{1}, t_{2}\right), t_{2} \neq t_{1}$. For each $n \geq 1$ let $\left(X^{n}(s): 0 \leq s<\infty\right)$ be a $T$-valued process adapted to a filtration $\left(\mathscr{A}_{s}^{n}\right)$ and such that the transition intensities

$$
R_{s}^{n}\left(\omega, t_{2}\right)=\lim _{\delta \downarrow 0} \delta^{-1} P\left(X_{s+\delta}^{n}=t_{2} \mid \mathscr{A}_{s}^{n}\right)(\omega)
$$

exist, for $t_{2} \neq X_{s}^{n}(\omega)$.

(More formally, $R_{s}^{n}$ is defined as satisfying the appropriate martingale characterization.)

Lemma 9. Suppose $X^{n}(0)=X(0)=x(0)$, deterministic. Define

$$
d_{s}^{n}\left(\omega, t_{1}, t_{2}\right)=\left|R_{s}^{n}\left(\omega, t_{2}\right)-R_{s}\left(t_{1}, t_{2}\right)\right| \text { if } X_{s}^{n}(\omega)=t_{1} \text { and } t_{2} \neq t_{1}
$$


and $=0$ otherwise. If, for each $s_{0}$ and $t_{1}, t_{2}$,

$$
\sup _{0 \leq s \leq s_{0}} d_{s}^{n}\left(\omega, t_{1}, t_{2}\right) \stackrel{p}{\rightarrow} 0 \text { as } n \rightarrow \infty
$$

then $\left(X_{s}^{n}\right) \stackrel{d}{\rightarrow}\left(X_{s}\right)$ in the sense of finite-dimensional distributions, and in particular

$$
X_{s}^{n} \stackrel{d}{\rightarrow} X_{s} \text { as } n \rightarrow \infty, s \text { fixed } .
$$

Sketch. Such a process $X^{n}$ can be described via the times $U_{i}^{n}$ and destinations $V_{i}^{n}$ of the $i$ th jump. It can be constructed in a canonical way from i.i.d. uniform r.v.'s $\left(U_{i}^{*}, V_{i}^{*}\right)$. One can show, under the hypotheses of the lemma, that these canonical versions (constructed for each $n$ and the limit process from the same $\left.\left(U_{i}^{*}, V_{i}^{*}\right)\right)$ converge a.s. to the limit canonical version as $n \rightarrow \infty$.

\subsection{Proof of Lemma 6}

We use a natural truncation idea. Fix a large integer $K$, and introduce a graveyard state $\Delta$. Define truncated processes

$$
\begin{aligned}
X(s) & =\mathscr{H}_{s} \text { if }\left|\mathscr{H}_{s}\right| \leq K \\
& =\Delta \text { if not } \\
X^{n}(s) & =\mathscr{F}_{n}(s) \text { if }\left|\mathscr{F}_{n}(s)\right| \leq K \\
& =\Delta \text { if not } .
\end{aligned}
$$

Because $\left|\mathscr{H}_{s}\right|<\infty$, to prove Lemma 6 it is enough to prove that for each fixed $K$ the truncated processes converge. For fixed $K$ our processes takes values in a finite state space (trees of size $\leq K$, and state $\Delta$ ), and so we shall prove convergence by verifying the hypothesis (23) of Lemma 9.

There are 5 types of possible transition $t_{1} \rightarrow t_{2}$, according as the $t_{i}$ have or do not have a distinguished vertex, or are $\Delta$.

Transition type 1. Suppose neither $t_{1}$ nor $t_{2}$ has a distinguished vertex. Quoting (12),

$$
R_{s}\left(t_{1}, t_{2}\right)=\sum_{\nu \in t} \sum_{\text {trees } x} 1_{\left(\left(t_{1}, v, x\right\rangle=t_{2}\right)} \pi_{s}(x)
$$

To calcualte the transition intensity for $X^{n}(s)$, we take the filtration generated by the entire random graph process $\left(\mathscr{G}\left(n, s^{\prime} /(n-1)\right), s^{\prime} \leq s\right)$. If two vertices are in different components at time $s$, the chance that an edge between them is created during $[s, s+d s]$ is $d s /(n-1-s)$. It follows that, on $\left\{X^{n}(s)=t_{1}\right\}$,

$$
R_{s}^{n}\left(\omega, t_{2}\right)=e_{1}+\frac{1}{n-1-s} \times \text { number of pairs }(v, i) \text { such that: }
$$

$v$ is a vertex of $t_{1}$;

$i$ is a vertex of $\mathscr{G}(n, s /(n-1))$ whose component, considered as a graph rooted at $i$, is a tree $x$, say;

$i$ is not in the component containing 1 ;

and $\left\langle t_{1}, v, x\right\rangle=t_{2}$. 
Here $e_{1}$ is an error term, incorporating the possibility that a transition from $t_{1}$ to $t_{2}$ might occur by $t_{1}$ being attached to some nontree component. We can rewrite the expression above as

$$
e_{1}+e_{2}+\sum_{v \in t} \sum_{\text {trees } x} 1_{\left(\left\langle t_{1}, v, x\right)=t_{2}\right)} C_{s}^{n}(x)
$$

where $e_{2}$ is another error term incorporating the possibility that the sum erroneously includes the component containing 1.

Now Lemma 7 implies that the sum in (25) converges to the sum in (24), because we need only consider trees $x$ of size at most $K$. The error term $e_{2}$ can be crudely bounded by $K^{2} /(n-s-1)$, which tends to 0 . The error term $e_{1}$ can be bounded by

$$
\frac{K}{n-1-s} \times \text { number of vertices in nontree components of size } \leq K
$$

and this tends to 0 by Lemma 7 .

Thus we have verified (23) for this type of transition.

Transition type 2. Suppose $t_{1}$ is a tree without a distinguished vertex, and $t_{2}$ is the same tree with vertex $v$, say, distinguished. (Of course there may be several $v$ 's which produce isomorphic $t_{2}$ 's, but then we just sum: so let us assume there is a unique $v$.) The limit process has transition rate

$$
R_{s}\left(t_{1}, t_{2}\right)=1-F(s)
$$

For the finite processes, we have: on $\left\{X^{n}(s)=t_{1}\right\}$,

$$
R_{s}^{n}\left(\omega, t_{2}\right)=\frac{1}{n-1-s} \times \text { number of vertices } i \text { such that: }
$$

$i$ is not in the component of $\mathscr{G}(n, s /(n-1))$ containing 1 ;

the size of component containing $i$ is at least $a(n)-\left|t_{1}\right|$.

As $n \rightarrow \infty$ this quantity is asymptotic to $A_{s}^{n, 1}$, in the notation of Lemma 8 with $a_{1}(n)=a(n)-\left|t_{1}\right|$, and then the assertion of Lemma 8 implies convergence of the transition intensities to the limit (26).

Transition type 3. Consider transitions from a tree $t_{1}$ without distinguished vertex to state $\Delta$. We prove convergence of transition intensities by a trick: by the previous results, it suffices to prove convergence for the total transition rate out of state $t_{1}$. But for the limit process, this total transition rate is $\left|t_{1}\right|$; and for the finite processes the total transition rate is

$$
\frac{1}{n-1-s}\left|t_{1}\right|\left(n-\left|t_{1}\right|\right)
$$

Transition type 4. Suppose both $t_{1}$ and $t_{2}$ have a distinguished vertex. The argument follows the argument for type 1 , except that the error term $e_{2}$ is absent.

Transition type 5 . Consider transitions from a tree $t_{\mathfrak{l}}$ with a distinguished vertex to state $\Delta$. We repeat the trick of considering instead the total transition rate out of state $t_{1}$. For the limit process, this total transition rate is

$$
\left|t_{1}\right| F(s)
$$


For the finite processes, the transition intensity is

$$
\frac{1}{n-1-s}\left|t_{1}\right| \times n-\text { size of component of } \mathscr{G}(n, s /(n-1)) \text { containing } 1 \text {. }
$$

Since $t_{1}$ has been given a distinguished vertex, the size of that component is at least $a(n)$. In the notation of Lemma 8 , the proportional size of the component is between $A_{s}^{n, 2}$ and $A_{s}^{n, 1}$, and so by the assertion of Lemma 8 the intensities above converge to those of (27).

\section{EXPLICIT CALCULATIONS WITH THE FRINGE}

\subsection{Proof of Proposition 2}

A key fact, proved later, for doing calculations with the limit $\mathscr{H}^{0}$ is

Lemma 10. $\operatorname{dist}\left(\mathscr{H}_{L}^{0} \mid L=s\right)=\operatorname{dist}\left(\mathscr{G}_{\hat{s}}\right)$, where $\hat{s}$ was defined at (10).

Since we know (13) the distribution of $L$, this lemma gives the distribution of $\mathscr{H}_{L}^{0}$, and the only issue is to take account of the evolution of $\mathscr{H}_{s}^{0}$ over $L<s<\infty$. Let $D(s), s \geq L$ be the degree of the root of $\mathscr{H}_{s}^{0}$. Lemma 10 implies

$$
\operatorname{dist}(D(L) \mid L=l) \text { is Poisson }(\hat{l})
$$

The chance that the degree increases by 1 during $[s, s+d s]$ is $F(s) d s$, and so

$$
\operatorname{dist}(D(\infty)-D(L) \mid L=l) \text { is Poisson }\left(\int_{l}^{\infty} F(s) d s\right)
$$

and this increment is conditionally independent of $D(L)$. Together with (28) and the definition of $\hat{s}$, we see

$$
\operatorname{dist}(D(\infty) \mid L=l) \text { is Poisson }(h(l))
$$

where

$$
h(x)=x F(x)+\int_{x}^{x} F(s) d s
$$

To reconcile this with the expression in Proposition 2, we must show $h(L) \stackrel{d}{=}$ $\Phi(U)$ for $U$ uniform on $[0,1]$. Now $h(x)=\int_{x}^{x} s F(d s)$, by integrating by parts. Writing $u=F(s)$,

$$
\begin{aligned}
h(x) & =\int_{0}^{F^{-1}(x)} F^{-1}(u) d u \\
& =\int_{0}^{F^{-1}(x)} \frac{\log 1 / u}{1-u} d u \text { by }(7) \\
& =\Phi\left(F^{-1}(x)\right)
\end{aligned}
$$


for $\Phi$ as stated in Proposition 2. Thus $h(L)=\Phi\left(F^{-1}(L)\right)$. But $F$ is the tail distribution function of $L$, so $F^{-1}(L)$ is uniform on $[0,1]$, and we are finished.

Note that

$$
\begin{aligned}
E D(\infty) & =E \Phi(U) \\
& =\int_{0}^{1} \Phi(u) d u \\
& =\int_{0}^{1} \int_{0}^{1} \frac{\log x}{x-1} 1_{(x<u)} d x d u \\
& =-\int_{0}^{1} \log x d x \\
& =1
\end{aligned}
$$

verifying the second assertion of Theorem 1 .

Numerical values are

$$
\begin{array}{cccccccc}
i & 0 & 1 & 2 & 3 & 4 & 5 & 6 \\
P(D(\infty)=i) & 0.408 & 0.324 & 0.171 & 0.068 & 0.022 & 0.006 & 0.001
\end{array}
$$

Remarks. In principle one can calculate the limit $\pi(t)$ for any finite tree $t$, but the results do not seem tractable. Similarly, one could attempt to study the distribution of size $\left|\mathscr{H}_{x}^{0}\right|$ of the fringe tree; it is easy to write down the generating function of the size $\left|\mathscr{H}_{L}^{0}\right|$ and a differential equation for the evolution of the generating function of $\left|\mathscr{K}_{s}^{0}\right|$ on $s>L$, but it is not clear (to the author!) how to extract useful information from this analysis. (Incidentally, general theory [1] says that the expected size $E\left|\mathscr{K}_{x}^{0}\right|=\infty$.)

Proof of Lemma 10. The lemma can be proved combinatorially from (14), but then seems mysterious. The following probabilistic argument explains why it is true.

Consider the Galton-Watson tree $\mathscr{G}_{s}$. We can describe the tree by describing the number $N$ of children of the root and the subtrees $\mathscr{G}_{s}^{i}$ rooted at each child. Obviously, $N$ has Poisson(s) distribution, and the $\mathscr{G}^{i}$ are i.i.d. copies of $\mathscr{G}_{s}$ independent of $N$. We want to condition on $\{L=s\}$. Each $\mathscr{G}_{s}^{i}$ can be regarded as being embedded in a process $\left(\mathscr{G}_{u}^{i}: 0 \leq u<\infty\right)$, an independent copy of the process of Section 2, which becomes infinite at time $L_{i}$ say. Conditioning on $\{L=s\}$ is conditioning on $\left\{\min _{i \leq N} L_{i}=s\right\}$. But a general fact (abstracted below) about Poisson distributions says that under this conditioning the number $N-1$ of other subtrees (other than the one with $L_{i}=s$ ) has Poisson $(s P(L>s)$ ) distribution, and the other subtrees are independent and distributed as $\mathscr{G}_{s}$ given $L>s$. Since $s P(L>s)=\hat{s}$ and $\operatorname{dist}\left(\mathscr{G}_{s} \mid L>s\right)=\operatorname{dist}\left(\mathscr{G}_{\xi}\right)$, and the tree formed by these other branches is $\mathscr{H}_{L}^{0}$, this is the assertion of the lemma.

The general fact cited above is as follows. Let $\left(\xi_{i}\right)$ be i.i.d. Let $N$ have Poisson $(\lambda)$ distribution, independent of the $\xi_{i}$. Let $f\left(\xi_{i}\right)$ be real-valued continuous. Conditional on $\min _{i \leq N} f\left(\xi_{i}\right)=s\left(=f\left(\xi_{i_{0}}\right)\right.$, say), we have

$N-1$ has Poisson $(\lambda P(f(\xi)>s))$ distribution

$\left(\xi_{i}, i \neq i_{0}\right)$ are independent and distributed as $\xi$ given $f(\xi)>s$.

This fact follows from the "independence under subdivision" property of the 
Poisson distribution, considering separately the $\xi$ 's with $f(\xi)<s$, with $f(\xi) \in$ $[s, s+d s]$ and with $f(\xi)>s+d s$.

\subsection{The Edge-weight at the Root}

For the application to minimum-weight spanning trees, we study a certain random time $J$ associated with the limit random tree-process $\left(\mathscr{H}_{s}\right)$. In words, $J$ is the time of attachment of the edge leading from the root towards the distinguished vertex. To say this precisely, let $\tau_{1}<\tau_{2}<\cdots$ be the times $s$ at which successive edges $E_{1}$, $E_{2}, \ldots$ are attached to the root of $\mathscr{H}_{s}$. Recall that $V^{*}$ is the distinguished vertex, and $L$ is the time at which it is attached. Then

$$
\begin{aligned}
& J=L \text { if } V^{*}=\text { root } \\
& J=\tau_{i} \text { if the path from root to } V^{*} \text { starts along edge } E_{i} .
\end{aligned}
$$

So $0<J \leq L$. To get an expression for the distribution of $J$, we return to the discription of the process $\left(\mathscr{H}_{s}\right)$ in Section 2 . It is clear that $\left(\tau_{1}, \tau_{2}, \ldots\right)$ are the times of successive events in a Poisson(1) process. Consider the time when (in the full process $\mathscr{G}_{s}$, rather then the restriction $\mathscr{H}_{s}$ ) the subtree attached via edge $E_{i}$ becomes infinite. This time can be represented as $\tau_{i} \vee L_{(i)}$, where the $L_{(i)}$ are distributed as $L$ and are independent as $i$ varies. One consequence of the representation is the distributional relation

$$
L \stackrel{d}{=} \min _{i} \tau_{i} \vee L_{(i)}
$$

which leads to an alternative derivation of the fundamental identity (6), which we restate in terms of $\bar{F}(s)=1-F(s)$ as follows. The distribution function

$$
\bar{F}(s)=P(L \leq s)=P\left(\left|\mathscr{G}_{s}\right|=\infty\right)
$$

satisfies $\bar{F}(s)=0$ on $0 \leq s \leq 1$ and

$$
1-\bar{F}(s)=\exp (-s \bar{F}(s)), \quad 1 \leq s<\infty
$$

So on $1<s<\infty$ we can specify $\bar{F}$ via its inverse function

$$
\bar{F}^{-1}(u)=\frac{-\log (1-u)}{u}, \quad 0<u<1 .
$$

Now in view of (31), the definition of $J$ becomes

$$
J=\tau_{I}, \quad \text { where } I=\arg \min _{i} \tau_{i} \vee L_{(i)} .
$$

We can now calculate

$$
\begin{array}{r}
P\left(x \leq \tau_{l} \leq x+d x, y \leq L_{l} \leq y+d y\right) \\
\quad=d x \bar{F}(d y) P\left(\min _{i} \tau_{i} \vee L_{i}>x \vee y\right) \\
\quad=d x \bar{F}(d y) \exp (-(x \vee y) \bar{F}(x \vee y)) \\
\quad=d x \bar{F}(d y)(1-\bar{F}(x \vee y)) \text { by }(32)
\end{array}
$$


where the second equality is obtained by viewing $\left(\tau_{i}, L_{i}\right)$ as a Poisson point process on the plane with rate $d x \vec{F}(d y)$. Integrating over $y$, we see that $J$ has density

$$
f_{J}(x)=\int_{1}^{x}(1-\bar{F}(x \vee y)) \bar{F}(d y)
$$

For $x \geq 1$ this becomes

$$
\begin{aligned}
f_{J}(x) & =1-\bar{F}^{2}(x)-\int_{x}^{x} \bar{F}(y) \bar{F}(d y) \\
& =1-\bar{F}^{2}(x)-\frac{1}{2}\left(1-\bar{F}^{2}(x)\right) \\
& =\frac{1}{2}\left(1-\bar{F}^{2}(x)\right) .
\end{aligned}
$$

And since $\bar{F}=0$ on $(0,1)$, the formula (33) is true for all $0 \leq x<\infty$.

\subsection{A Calculus Exercise}

Here is the argument for (4). Put $a=1 / \rho$ for convenience. Note that the substitution $u=1-\bar{F}(x)$ makes $x=\frac{\log (1 / u)}{1-u}$ and $\bar{F}(d x)=-d u$.

$$
\begin{aligned}
\int_{0}^{x} x^{a} f,(x) d x & =\int_{0}^{x} x^{a+1} \bar{F}(x) \bar{F}(d x) \text { by parts } \\
& =\int_{1}^{x} x^{a+1} \bar{F}(x) \vec{F}(d x) \\
& =\int_{0}^{1} \log ^{a+1}(1 / u)(1-u)^{-a} d u \text {, putting } u=1-\bar{F}(x) \\
& =\int_{0}^{1} \log ^{a+1}(1 / u) \sum_{i=0}^{x} a+i-1 C_{i} u^{i} d u \\
& =\int_{0}^{x} s^{a+1} \sum_{i=0}^{x} a+i-1 C_{i} e^{-s(i+1)} d s, \text { putting } u=e^{-s} \\
& =\sum_{i=0}^{x} a+i-1 \\
& =a(a+1) \frac{(a+1) !}{(i+1)^{a+2}} \\
& \sum_{i=0}^{x} \frac{(a+i-1) !}{i !(i+1)^{a+2}} .
\end{aligned}
$$

Here we wrote ${ }_{n} C_{m}$ for " $n$ choose $m$."

\subsection{Minimum-weight Spanning Trees}

Proof of Proposition 3. Regard $\mathscr{T}_{n}$ as a random tree in which each edge has "weight" equal to the time at which it was added, in the process $\left(\mathscr{T}_{n}(s)\right)$. Define $J_{n}(1)$ to be the weight of the edge $\left(E_{n}(1)\right.$, say) at vertex 1 leading towards the 
distinguished edge $\left(V_{n}^{*}, V_{n}^{* *}\right)$. Thus $J_{n}$ is analogous to the definition (30) of $J$, with $L_{n}$ in place of $L$. Repeat this construction for each vertex $v=1, \ldots, n$ to obtain a weight $J_{n}(v)$ of an edge $E_{n}(v)$ incident at $v$. From Lemma 5 , in which we may regard the edges being weighted, we have

$$
J_{n}(1) \stackrel{d}{\rightarrow} J
$$

It is also easy to see

$$
P\left(E_{n}(1)=E_{n}(v) \text { for some } v \neq 1\right) \rightarrow 0 \text { as } n \rightarrow x .
$$

By symmetry, $J_{n}(v) \stackrel{d}{=} J_{n}(1)$ for each $v$. Let $V_{n}$ be a uniform random vertex of $\mathscr{T}_{n}$. Then $J_{n}\left(V_{n}\right) \stackrel{d}{\rightarrow} J$. But (34) implies that the mean proportion of edges of $\mathscr{T}_{n}$ which occur exactly once in $\left(E_{n}(v) ; v=1, \ldots, n\right)$ tends to 1 . So asymptotically, $J_{n}\left(V_{n}\right)$ behaves as the weight $\hat{\theta}_{n}$ of a uniform random edge of $\mathscr{T}_{n}$. This establishes

$$
\hat{\theta}_{n} \stackrel{d}{\rightarrow} J
$$

This $\hat{\theta}_{n}$ is the weight of a uniform random edge of the minimum spanning tree, in the special case where the edge-weights $\hat{\eta}^{(n)}$ are distributed uniformly on $(0, n-1)$. But the case of general edge-weights $\eta^{(n)}$ can be constructed from the special case by simply putting $\eta^{(n)}=G_{n}^{-1}\left(\hat{\eta}^{(n)} /(n-1)\right)$, and so the edge-weight distribution $\theta_{n}$ in the general case relates to the special case via

$$
\theta_{n}=G_{n}^{-1}\left(\hat{\theta}_{n} /(n-1)\right)
$$

Now Proposition 3 follows from (35).

Proof of Corollary 4. What we must prove is $E \theta_{n} \sim E G_{n}^{-1}(J / n)$, that is

$$
E G_{n}^{-1}\left(\hat{\theta}_{n} /(n-1)\right) \sim E G_{n}^{-1}(J / n) .
$$

Now $J$ has support $[0, \infty)$ and $G_{n}^{-1}$ is positive nondecreasing: using (35), standard integration theory says that a sufficient condition for (36) is

$$
\frac{G_{n}^{-1}\left(\hat{\theta}_{n} / n\right)}{G_{n}^{-1}\left(x_{0} / n\right)} \text { is uniformly integrable }
$$

for some $0<x_{0}<\infty$. Using hypothesis (2), it is sufficient to show that there exist $\beta>0, B<\infty$ such that

$$
P\left(\hat{\theta}_{n}>s\right) \leq B e^{-\beta s}, \quad s \geq 2, n \geq 2 .
$$

Recall the construction above of edges $E_{n}(v)$ with weights $J_{n}(v)$. Because each edge of $\mathscr{T}_{n}$ can occur at most twice in $\left(E_{n}(v) ; v=1, \ldots, n\right)$,

$$
P\left(\hat{\theta}_{n} \in \cdot\right) \leq \frac{2 n}{n-1} P\left(J_{n}\left(V_{n}\right) \in \cdot\right)=\frac{2 n}{n-1} P\left(J_{n}(1) \in \cdot\right) .
$$


Now $J_{n}(1) \leq L_{n}$, where $L_{n}$ is the time at which vertex 1 gets connected to the giant component. So it is enough to show a bound of the form

$$
P\left(L_{n}>s\right) \leq B e^{-\beta s}, \quad s \geq 2, n \geq 2 .
$$

But this is easy, e.g., because

(i) $P\left(L_{n}>s\right) \rightarrow P(L>s)=\vec{F}(s)<1$ as $n \rightarrow \infty$, $s>1$ fixed;

(ii) the map $s \rightarrow P\left(L_{n}>s\right)$ is submultiplicative, for fixed $n$.

\section{MISCELLANEOUS COMMENTS}

\subsection{Convergence in Probability}

Summarizing Corollary 4 as

$$
E W_{n} \sim w_{n}
$$

we would expect the stronger result giving convergence in probability

$$
W_{n} / w_{n} \stackrel{p}{\rightarrow} 1
$$

to be true; and this was known in the special cases. Similarly, writing $\Delta_{n}(i)$ for the empirical proportion of vertices of $\mathscr{T}_{n}$ which have degree $i+1$, Proposition 2 says

$$
E \Delta_{n}(i) \rightarrow P(D(\infty)=i), \quad i \geq 0
$$

and one would expect the stronger result

$$
\Delta_{n}(i) \stackrel{p}{\rightarrow} P(D(\infty)=i) .
$$

An abstract way of proving such extensions was developed in [1]. By verifying an "extremality" condition on the asymptotic fringe distribution $\pi$, one can deduce that convergence in probability is an immediate consequence of convergence of expectations for all local functionals. The required verification for our $\pi$ is sketched in [1], and this implies $(38,39)$ in particular.

\subsection{The Dual Tree}

There is another model which is, loosely speaking, dual to our model. Start with the complete graph on $n$ vertices. At each stage, pick and delete an edge chosen uniformly from the set of edges whose deletion would not disconnect the graph. This gives a random graph $\mathscr{T}_{n}^{*}$ which (for $n \geq 5$ ) is different from $\mathscr{T}_{n}$. For example, for the star $t_{5}$ on 5 vertices centered at 1 ,

$$
P\left(\mathscr{T}_{5}=t_{5}\right)=\frac{1}{105}, \quad P\left(\mathscr{T}_{5}^{*}=t_{5}\right)=\frac{46}{45} \times \frac{1}{105}
$$


Here we briefly outline an argument that this dual tree has the same limit fringe distribution $\pi$ as the original tree.

Consider again the random graph process $\mathscr{G}(n, s /(n-1))$, but now regard time $s$ as decreasing from $\infty$ to 0 . Associate with it another graph process $\mathscr{H}_{n}(s)$ with the properties

$\mathscr{H}_{n}(s)$ coincides with $\mathscr{G}(n, s /(n-1))$ on components of $\mathscr{G}(n, s /(n-1))$.

$\mathscr{H}_{n}(s)$ is connected.

Specifically, when an edge of $\mathscr{G}(n, s /(n-1))$ is deleted (as $s$ decreases), the same edge in $\mathscr{H}_{n}(s)$ is deleted, provided this would not disconnect the graph. Then $\mathscr{H}_{n}(0)$ is the "dual random tree" under consideration.

The component containing vertex 1 is disconnected from the giant component of $\mathscr{G}(n, s /(n-1))$ at time $L_{n}$, the same as the connection time in Section 3. After disconnecting, 1 is in a component $\mathscr{G}\left(n, \frac{L_{n}-}{n-1}\right) \subseteq \mathscr{H}_{n}\left(L_{n}-\right)$. As $n \rightarrow \infty, \mathscr{G}\left(n, \frac{L_{n}-}{n-1}\right)$ becomes a tree-component, and $\mathscr{H}_{n}\left(L_{n}-\right)$ consists of $\mathscr{G}\left(n, \frac{L_{n}-}{n-1}\right)$ and other tree-components $\mathscr{G}\left(n, \frac{s_{i}-}{n-1}\right)$ which disconnected from the giant component in the random graph process at times $S_{i}>L_{n}$. The fringe subtrees we study are representable as $\mathscr{H}_{n}^{0}\left(L_{n}-\right)$, where ${ }^{0}$ denotes "delete branch containing vertex at which disconnection from giant component occurs." Asymptotically, $\mathscr{G}\left(n, \frac{L_{n}-}{n-1}\right)$ behaves exactly as $\mathscr{H}_{L}^{0}$ in the original model. And the way that other components of $\mathscr{G}(n, s /(n-1))$ disconnect from $\mathscr{G}\left(n, \frac{L_{n}-}{n-1}\right)$ as $\infty>s \downarrow L_{n}$ is asymptotically the same as the way that in the original model $\mathscr{H}_{s}^{0}$ grows on $L_{n}<s \uparrow \infty$.

\subsection{Global Structure}

Theorem 1 does not give information about "global" properties of the trees, such as height and diameter. Global asymptotics for the uniform random tree on $n$ vertices were treated in [2]. I conjecture that the main result there (Theorem 8: convergence to the compact continuum tree) holds also for the model under consideration here. This would imply that the known asymptotics for height, diameter, etc. for the uniform random tree can be applied also to $\mathscr{T}_{n}$. But this seems difficult to prove.

\section{REFERENCES}

[1] D.J. Aldous, Asymptotic fringe distributions for general famileis of random trees, Ann. Appl. Probab., to appear.

[2] D.J. Aldous, The continuum random tree I, Ann. Probab., to appear.

[3] F. Avram and D. Bertsimas, The Minimum Spanning Tree Constant in Geometric Probability and under the Independent Model: A Unified Approach, Tech. Rep., Massachusetts Institute of Technology, 1990.

[4] B. Bollobás, Random Graphs, Academic Press, London, 1985.

[5] B. Bollobás and I. Simon, On the expected behavior of disjoint set union algorithms, in Proc. 17th STOC, 1985, pp. 224-231.

[6] A.M. Frieze, On the value of a random minimum spanning tree problem, Discrete Appl. Math., 10, 47-56 (1985). 
[7] D.E. Knuth and A. Schonhage, The expected linearity of a simple equivalence algorithm, Theor. Computer Sci., 6, 281-315 (1978).

[8] E.A. Timofeev, On finding the expected length of a random minimal tree, Th. Probab. Appl., 32, 361-365 (1987).

[9] A. Yao, On the average behavior of set merging algorithms. in Proc. 8th STOC, 1976, pp. 192-195.

Received December 11, 1989 